\title{
Common origin of both coronary arteries from LSV
}

\author{
Avanish Kumar', Md. Jawed Akhtar', Lalit Mohan² \\ ${ }^{1}$ Department of Anatomy, ${ }^{2}$ Department of Pharmacology, Indira Gandhi Institute of Medical Sciences, Sheikhpura, Patna
}

\section{A B STRA C T}

During routine dissection of a male cadaver aged about 60 years in dept. of Anatomy at IGIMS Patna a rare anomalous single coronary artery has been detected. In this case a single trunk is taking origin from LSV. Both the coronary arteries are arising from this trunk. Heart's shape, size and weight are within normal range. On gross examination no other major congenital malformation and pathological changes is detected. In addition to this, further during dissection in other region of the body no other major vascular malformation is found.

Key words: SCA (Single coronary artery), LSV (Left sinus of Valsalva), Ostia, LCA (Left Coronary artery), RCA (Right coronary artery), LAD (Left anterior descending artery), LCx (Left circumflex)

\section{CASE REPORT}

On examination of above-mentioned heart we find the left and right coronary arteries have common origin, which is from Left sinus of Valsalva (LSV) (Figure 1). There is single ostium placed normally below the Sino-tubular junction at the dilated portion of sinus. Both in anterior \& right posterior (RSV) sinuses, there is no ostia. Common trunk just after originating from LSV, divide into RCA \& LCA. Almost at the same place LAD and LCx come out from LCA (Figure 2).

RCA is taking course behind the Aorta and reaching adjacent to RSV. From here it is passing forward between the root of the pulmonary trunk and right auricle. Then it is passing down into the atrioventricular groove (or sulcus) towards the crux (a point on the diaphragmatic surface of heart where right AV groove, Lt AV groove \& posterior interventricular groove come together). The first branch of RCA is right conus artery (arteria coni arteriosi).

Both the LAD \& LCx artery is running between the pulmonary trunk and the left atrial auricle, emerging into the atrioventricular groove. Later on LAD \& LCx took the normal course and branching pattern.

LCA is distinctly thicker and has larger lumen. The size of ostium in LSV is also remarkably more in comparison to a normal one.
The relationship between Aorta and Pulmonary Artery is normal. There is no other gross cardiac anomaly.

\section{DISCUSSION}

Normally the two major coronary arteries arise from two of the three sinuses of Valsalva, accordingly the sinuses has also been named as right coronary and left coronary sinus. Many authors have reviewed and reported anomalous origin of coronary arteries. Isolated single coronary artery is a rare congenital anomaly occurring in approximately $0.024 \%$ of

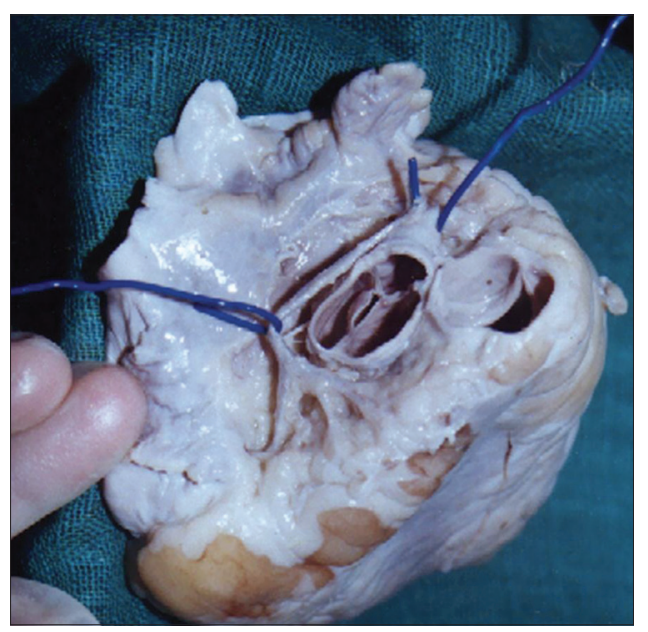

Figure 1: Photograph of the heart studied 


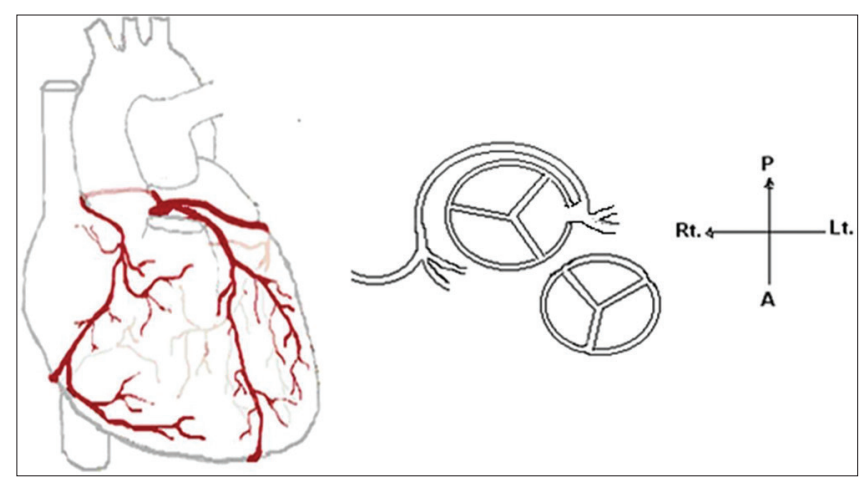

Figure 2: Single coronary artery arising from LSV: A diagrammatic illustration of the case. It also shows the branching patterns of LCA \& RCA, which are almost normal i.e. showing no anomaly

the population. ${ }^{1}$ The incidence of all coronary anomalies ranges from $0.3 \%$ to $1.2 \%$ \& Anomalous origin of RCA from LSV is reported to constitute from $6 \%$ to $27 \%$ of all coronary anomalies. ${ }^{2}$ An Angiographic classification of SCA pattern has been suggested by lipton et al. ${ }^{3}$

It may be hypothesized that an anomalous coronary arterial system should have different flow pattern with changed (higher or lower) flow rates, combined with a different set of pathophysiological reaction on the vessel wall. A combination of higher proximal flow rates and lower distal flow is expected which could result in a hemodynamic pattern of localized turbulence. This may lead to accelerated atherosclerosis. Many authors have studied regarding chance of developing atherosclerosis in anomalous coronary anatomy. A common consensus is that anomalous origin of coronary artery does not predispose it for developing atherosclerosis. Here it is worth mentioning the study of Melvin D Chetlin et $\mathrm{al}^{2}$ who published a list of 18 patients with both coronary arteries arising from left sinus of Valsalva. All died of known, unrelated causes. In the above-mentioned case also at the age 60 years there no clear cut coronary blockage is found on examination of coronary lumen.

The course of artery after taking origin from common trunk has much clinical significance. When it passes between the Aorta and Pulmonary trunk it may lead to sudden death. As have been studied by cheitlin MD et al when both coronary arteries arising from 'anterior sinus of Valsalva' have much clinical significance \& may cause sudden death ${ }^{2}$. In these cases mainly the course of artery is responsible for sudden death. In case of SCA where the vessel does not course between the aorta and pulmonary trunk, arteries are not vulnerable to acute angulations. Therefore, a single coronary artery is often a benign coronary anomaly, in which sudden death is a rare complication.

A critical proximal stenosis in a single coronary artery may cause severe coronary insufficiency since a large area of myocardial perfusion is jeopardized. Another explanation for severe coronary insufficiency is the inability to recruit a competent collateral vascular network from an absent contralateral artery. The aforementioned arguments emphasize the potential increased periprocedural risk during angioplasty.

\section{REFERENCES}

1. Desmet W, Vanhaecke J, Vrolix M, Van de Werf F, Piessens J, Willems $\mathrm{J}$, et al. Isolated single coronary artery: a review of 50,000 consecutive coronary angiographies. Eur Heart J 1992;13(12):1637-1640.

2. Cheitlin MD, De Castro CM and McAllister HA. Sudden death as a complication of anomalous left coronary origin from the anterior sinus of Valsalva, a not-so-minor congenital anomaly. Circulation 1974; 50:780-787.

3. Lipton MJ, Barry WH, Obrez I, Silverman JF and Wexler L. Isolated single coronary artery: diagnosis, angiographic classification, and clinical significance. Radiology 1979;130(1):39-47. 